\title{
Static Inter-Cell Interference Coordination Techniques for LTE Networks: A Fair Performance Assessment
}

\author{
David González G, Mario García-Lozano, Silvia Ruiz, and Joan Olmos * \\ Universitat Politecnica de Catalunya (UPC), \\ C/ Esteve Terradas, 7 - 08860 Castelldefels, Spain \\ [david.gonzalez.gonzalez, mario.garcia-lozano] @upc.edu
}

\begin{abstract}
This paper focuses in the analysis of $100 \%$ static and distributed inter-cell interference coordination techniques in the context of LTE networks. Several methods have been modeled and studied with the aim of deriving practical radio planning rules based on the joint effect of operational parameters and thresholds. The investigation places special emphasis on the efficiency vs. fairness tradeoff. Several metrics have been detected as interesting to allow not only the performance measurement from different point of view, but also to look at the effectiveness in the utilization of resources. Results show that similar levels of spectral efficiency can be achieved by means of a proper and accurate network tuning. On the other hand, interesting second order differences appear due to some inherent features of each approach. These can be exploited depending on the particular network operator needs.
\end{abstract}

\section{Introduction}

In the context of mobile communications, the availability of new services and mobile applications jointly with the increasingly powerful terminals run up the need of higher data rates and to adequate the levels of quality of service for the users. In order to fulfill such expectations, mobile operators are continually optimizing and upgrading their networks. The Long Term Evolution (LTE) of the Universal Mobile Telecommunications System (UMTS) along with WiMAX seems to be the path to follow in a very short term. Both LTE and and WiMAX employ Orthogonal Frequency Division Multiple Access (OFDMA) as the radio access technology for the downlink because of its suitability and advantages in the radio channel. One of the most important is the intrinsic orthogonality provided by OFDMA to the users within a cell, which translates into an almost null level of intra-cell interference. Therefore, inter-cell interference is the limiting factor when high reuse levels are intended. In this case, cell-edge users are specially susceptible to the effects of inter-cell interference. Hence, their radio channel is much worse than users close to the base station and so, unless more

\footnotetext{
* This work has been funded through the project TEC2008-06817-C02-02 (Spanish Industry Ministry).
} 
resources are assigned to them, fairness among users is jeopardized. Unfortunately, this is at the cost of reducing the spectral efficiency in the cell area. It is clear that a tradeoff between efficiency and fairness exists.

Within the 3GPP, several alternatives have been proposed [1], (a) inter-cell interference coordination (ICIC), (b) randomization and (c) cancelation. This work focuses on inter-cell interference coordination techniques, particularly in static and distributed approaches, that means configurations that are adjusted during the radio planning process.

Broadly speaking, the main target of any ICIC strategy is to determine the resources (bandwidth and power) available at each cell at any time. Then (and typically), an autonomous scheduler assigns those resources to users. Thus, from the Radio Resource Control perspective, there are two kind of decisions: (a) which resources will be allocated to each cell? and, (b) which resources will be allocated to each user?. Clearly, the temporality of such decisions is quite different. Whereas resources to users allocation is in the order of milliseconds, the allocation of resources to cells take much longer periods of time or can be fixed. In fact, this work deals with this last case.

Static ICIC schemes are attractive for operators since the complexity of their deployment is very low and there is not need for new extra signaling out of the standard. Static ICIC mostly relies on the fractional reuse concept. This means that users are categorized according to their Signal-to-Noise-plus-Interference Ratio (SINR), that means basically according to their inter-cell interference, and different reuse factors are applied to them, being higher at regions with more interference, mostly outer regions of the cells. The total system bandwidth is divided into sub-bands which are used by the scheduler accordingly.

Typically, static strategies follow 2 approaches:

- Apply different reuse factors to inner and outer users, being lower in the first case. In this sense, pioneer references can be found in $[2,3,4]$ in which reuse factor 1 is applied to users in the central part of the cell, and a factor $>1$ to the users in the outer part. Some refinements and extensions were done in [5] and a comparison of these proposals in terms of spectral efficiency was presented in [6]. A formal analysis of fractional reuse can be found in [7].

- Reuse factor 1 is applied to the whole band but less power is devoted to inner users to reduce inter-cell interference. The authors in $[8,9]$ compute the optimum power levels so that the system throughput is maximized. Also, the optimization of static power levels were addressed analytically by the authors in [9].

The contribution of this work is to present a fair comparison among static ICIC schemes highlighting the role of the different elements affecting the efficiency vs. fairness tradeoff. To to do this, we evaluate an extended set of metrics which help us better understand the strengths and weaknesses of each method and we close the analysis providing some recommendations of practical interest based on the findings.

The paper is organized as follows, Section 2 provides the description of the static ICIC schemes we have considered and their corresponding configurations. 


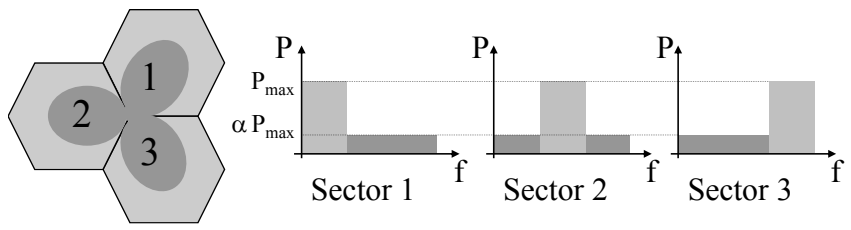

(a) ICIC S1.

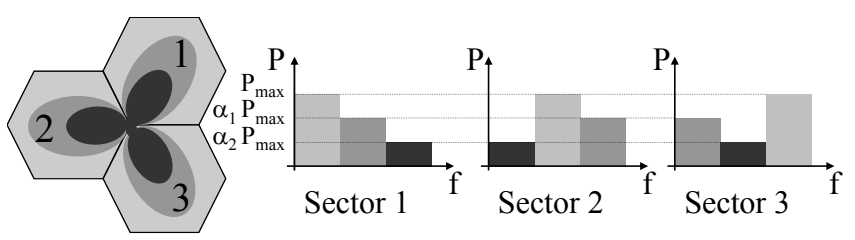

(b) ICIC S2.

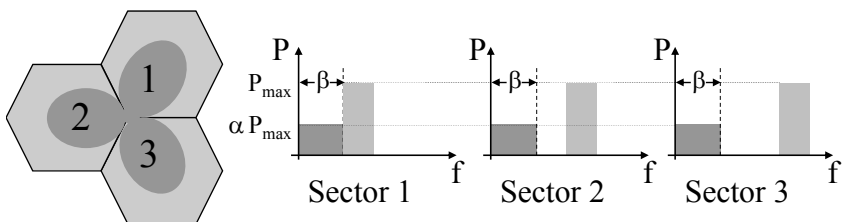

(c) ICIC S3.

Fig. 1. Classic power profiles in static ICIC schemes.

Next, Section 3 explains the methodology, this is followed by the analysis of results in Section 4. Finally, conclusions and other remarks close the paper in Section 5 .

\section{Description of ICIC strategies}

In this section, we present a detailed description of the static ICIC strategies we have considered for this study. The section finishes with the list of the performance metrics employed to asses the performance of those strategies.

\subsection{Static ICIC.}

As we commented before, in static ICIC schemes, the resources allocated to each cell do not change along time. They are computed and evaluated during the radio planning process and only long-term readjustments are performed during the operation of the network. The set of sub-carriers and the power levels allocated to them is fixed for each cell. A generic representation of the power profiles corresponding to each scheme is shown in Fig. 1.

As previously stated, these strategies mainly rely on a classification of the users based on the average SINR. Thus, in general, the group to which each user 
Table 1. Details of static ICIC schemes.

\begin{tabular}{llll}
\hline ICIC scheme & Sub-bands & Bandwidth $(\%)$ & User class \\
\hline \multirow{2}{*}{ S1 } & 1 & 33 & Inner \\
& 2 & 66 & Outer \\
\hline \multirow{2}{*}{ S2 } & 1 & 33 & Inner \\
& 2 & 33 & Centrals \\
& 3 & 33 & Outer \\
\hline \multirow{3}{*}{ S3 } & 1 & $\beta \cdot 100$ & Inner \\
\cline { 2 - 4 } & 2 & & \\
& 3 & $(1-\beta) \frac{1}{3} \cdot 100$ & Outer \\
\hline
\end{tabular}

belong highly depends on the position of the user within the cellular layout. Two possible criteria have been defined because the choice of the threshold to classify users has an immediate impact on the scheduler decisions and so on the system performance, as later is shown:

1. Class Proportionality: SINR thresholds are selected so that each class has the same average number of users.

2. Bandwidth Proportionality: The threshold guarantees that the number of users is proportional to its allocated bandwidth.

ICIC S1: In this scheme, we can observe from Fig. 1a that the design is done to guarantee a reuse factor 3 for outer users, while the inner ones have a lower value. In this case, inter-cell interference is also inter-class because of the assignment of bands among different cells. In order to control the amount of interference received by cell-edge users, low power is used in the bands to be used in the central area of the cell. This power is controlled by the parameter $\alpha$, which is the only tunable factor in this schema. In order to evaluate the impact of a poorly adjusted value, different cases have been considered in the analysis, these are shown in Table 2.

ICIC S2: This case is a natural extension of the previous scheme in order to study the effect of a more accurate classification of users. In this case we have three classes instead of two. Table 3 shows the different configurations. The analysis of this case is similar to the one for ICIC S1.

ICIC S3: As in ICIS S1, two different classes are also considered, however the novelty here is that inter-class interference is removed completely. In other words, each class has exclusive use of its bandwidth. This is important because the performance in terms of throughput and fairness becomes independent of $\alpha$ 
Table 2. Configurations considered for ICIC S1.

\begin{tabular}{llll}
\hline Config. Id. & $\alpha$ & $\begin{array}{l}\text { Classification } \\
\text { criterion }\end{array}$ & $\begin{array}{l}\text { Associated SINR } \\
\text { Threshold [dB] }\end{array}$ \\
\hline S1.a & 0.12 & & \\
\hline S1.b & 0.37 & BW Prop. & 0.35 \\
\hline S1.c & 0.75 & & \\
\hline S1.d & 1.00 & & \\
\hline S1.e & 0.12 & & \\
\hline S1.f & 0.37 & Class Prop. & \\
\hline S1.g & 0.75 & & \\
\hline S1.h & 1.00 & & \\
\hline
\end{tabular}

Table 3. Configurations considered for ICIC S2.

\begin{tabular}{lccll}
\hline Config. Id. & $\alpha_{1}$ & $\alpha_{2}$ & $\begin{array}{l}\text { Classification } \\
\text { criterion }\end{array}$ & $\begin{array}{l}\text { Associated SINR } \\
\text { Threshold [dB] }\end{array}$ \\
\hline S2.a & 1.00 & 1.00 & Class Prop. & $\{0.35,5\}$ \\
\hline S2.b & 0.66 & 0.33 & & \\
\hline S2.c & 0.5 & 0.25 & & \\
\hline
\end{tabular}

since the SINR does not depend on the transmitted power, equal for all cells, as long as the inter-cell interference level is greater enough than the noise floor. The parameter $\beta$ controls the width of the band allocated to inner users, hence it also determines the bandwidth available for outer ones. For this reason, it became necessary to evaluate this scheme for additional thresholds of SINR. Table 4 shows the different configurations considered for this strategy.

\subsection{Comparison of strategies. Performance Metrics.}

In order to evaluate the static ICIC strategies properly, a set of metrics have been taken into account. These allow to look at the performance from several perspectives. Many contributions do not take into account the fairness in their analysis. Moreover, very often a tradeoff oriented analysis is also missing. In this work, the main target is to provide a comprehensive evaluation of these ICIC strategies in which this tradeoff (fairness vs. efficiency) can be clearly understood. Table 5 lists the set of metrics. Note that a Physical Resource Block (PRB) is the minimum bandwidth the scheduler can assign to one single user. 
Table 4. Configurations considered for ICIC S3. $\alpha=0.5$.

\begin{tabular}{llll}
\hline Config. Id. & $\beta$ & $\begin{array}{l}\text { Classification } \\
\text { criterion }\end{array}$ & $\begin{array}{l}\text { Associated SINR } \\
\text { Threshold }[\mathrm{dB}]\end{array}$ \\
\hline S3.a & 0.10 & Class Prop. & 2.40 \\
S3.b & 0.25 & Class Prop. & 2.40 \\
S3.c & 0.25 & - & 3.95 \\
S3.d & 0.25 & BW Prop. & 7.05 \\
S3.e & 0.25 & - & 12.7 \\
S3.f & 0.40 & Class Prop. & 2.40 \\
S3.g & 0.40 & BW Prop. & 3.95 \\
S3.h & 0.49 & Class Prop. & 2.40 \\
\hline
\end{tabular}

Table 5. Performance metrics.

\begin{tabular}{ll}
\hline Metric & Units \\
\hline Spectral efficiency & $\frac{\mathrm{bps}}{\mathrm{Hz} \cdot \mathrm{km}^{2}}$ \\
\hline Average cell rate & $\mathrm{bps}$ \\
\hline Average user rate & $\mathrm{bps}$ \\
\hline Weakest user rate & $\mathrm{bps}$ \\
\hline Fairness (Jain's Index $[10]):$ Assuming that fair means equal throughput, N/A \\
this metric measures if $n$ users are receiving equal treatment. The result \\
ranges from $1 / n$ (worst case) to 1 (best case). \\
\hline Payload per PRB: Effectiveness in the bandwidth usage. \\
\hline Average transmitted power per cell. & $\frac{\mathrm{bits}}{\mathrm{PRB}}$ \\
\hline Payload per Watt: Effectiveness in the power usage. & $\mathrm{W}$ \\
\hline
\end{tabular}

\section{Methodology}

We consider the downlink of a cellular network composed by 57 cells in a regular tri-sectorial layout with an inter-site distance of $1.5 \mathrm{~km}$. The OFDMA configuration follows the setting establish by the LTE standard $[11,12,13]$. Specifically, the system has $100 \mathrm{PRB}$ available for the users $(18 \mathrm{MHz}, 1200$ sub-carriers of $15 \mathrm{kHz}$ ). Transmission time intervals of $1 \mathrm{~ms}$ containing 10 OFDMA symbols are considered. The total available power at each cell is $43 \mathrm{dBm}$. We studied and quantified the performance of the different strategies by means of a semistatic system level simulator. In particular, independent snapshots are generated and run for short periods of time $(18 \mathrm{~ms})$. Statistics were collected from the 21 central cells to avoid border effects. 3GPP urban macro-cellular and ITU Extended Typical Urban (ETU) have been considered as propagation and channel models respectively. $8 \mathrm{~dB}$ log-normal shadowing is applied following the model 
Table 6. Overall comparison.

\begin{tabular}{|c|c|c|c|c|c|c|c|c|}
\hline Id. & $\begin{array}{l}\text { Spectral } \\
\text { efficiency } \\
{\left[\frac{\mathrm{bps}}{\mathrm{Hz} \cdot \mathrm{km}^{2}}\right]}\end{array}$ & $\begin{array}{l}\text { Cell } \\
\text { rate } \\
{[\mathrm{Mbps}]}\end{array}$ & $\begin{array}{l}\text { User } \\
\text { rate } \\
{[\mathrm{Mbps}]}\end{array}$ & $\begin{array}{l}\text { Weakest } \\
\text { user rate } \\
{[\mathrm{kbps}]}\end{array}$ & $\begin{array}{l}\text { Fairness } \\
{[\text { J. index }]}\end{array}$ & $\begin{array}{l}\text { Payload } \\
\text { per PRB } \\
{\left[\frac{\text { bits }}{\text { PRB }}\right]}\end{array}$ & $\begin{array}{l}\text { Power } \\
{[\mathrm{W}]}\end{array}$ & $\begin{array}{l}\text { Payload } \\
\text { per Watt } \\
{\left[\frac{\mathrm{kb}}{\mathrm{W}}\right]}\end{array}$ \\
\hline S1.a & 2.19 & 25.60 & 1.91 & 130 & 0.62 & 257.9 & 19.6 & 23.5 \\
\hline S1.b & 2.33 & 27.18 & 2.03 & 130 & 0.64 & 273.8 & 19.7 & 24.8 \\
\hline S1.c & 2.39 & 27.92 & 2.09 & 100 & 0.63 & 281.3 & 19.8 & 25.4 \\
\hline S1.d & 2.42 & 28.25 & 2.11 & 50 & 0.62 & 284.6 & 19.8 & 25.7 \\
\hline S1.e & 2.56 & 29.88 & 2.23 & 130 & 0.62 & 299.1 & 19.9 & 27.0 \\
\hline S1.f & 2.67 & 31.21 & 2.33 & 120 & 0.59 & 312.3 & 19.9 & 28.2 \\
\hline S1.g & 2.72 & 31.76 & 2.37 & 80 & 0.56 & 317.9 & 19.9 & 28.7 \\
\hline S1.h & 2.74 & 32.00 & 2.39 & 50 & 0.55 & 320.2 & 19.9 & 28.9 \\
\hline S2.a & 2.00 & 23.44 & 1.75 & 50 & 0.63 & 238.15 & 19.64 & 21.5 \\
\hline S2.b & 1.85 & 21.57 & 1.61 & 110 & 0.67 & 219.18 & 19.57 & 19.84 \\
\hline $\mathrm{S} 2 . \mathrm{c}$ & 1.80 & 21.00 & 1.57 & 130 & 0.67 & 213.36 & 19.32 & 19.32 \\
\hline S3.a & 1.38 & 16.12 & 1.20 & 30 & 0.50 & 403.67 & 19.91 & 14.57 \\
\hline S3.b & 1.74 & 20.32 & 1.52 & 90 & 0.71 & 406.87 & 19.92 & 18.36 \\
\hline S3.c & 1.88 & 21.95 & 1.64 & 80 & 0.74 & 439.19 & 19.94 & 19.81 \\
\hline S3.d & 2.09 & 24.46 & 1.83 & 70 & 0.69 & 490.63 & 19.91 & 22.11 \\
\hline S3.e & 2.28 & 26.70 & 2.00 & 70 & 0.47 & 555.10 & 19.45 & 24.71 \\
\hline S3.f & 2.11 & 24.63 & 1.84 & 60 & 0.71 & 410.77 & 19.93 & 22.24 \\
\hline S3.g & 2.27 & 26.48 & 1.98 & 50 & 0.66 & 441.48 & 19.94 & 23.90 \\
\hline S3.h & 2.33 & 27.21 & 2.03 & 40 & 0.66 & 412.52 & 19.93 & 24.57 \\
\hline
\end{tabular}

proposed in [14] with a correlation coefficient between cells equal to 0.5 . It is important to note that achievable rates were computed taking into account the instantaneous channel conditions and according to the adaptive modulation and coding used in LTE, as specified in [15]. This mapping has been done using the link abstraction model based in mutual information at modulation symbol level [16], which outperforms the classic Effective Exponential SINR model because it is able to predict the BLER with higher accuracy, particularly for higher order modulations, such as 64-QAM.

\section{Numerical Results}

Table 6 shows the results obtained for the experiments defined in Table 2, 3 and 4. Nevertheless, for comparison purposes, some of the results are shown graphically in Fig. 2.

Figures $2 \mathrm{a}$ and $2 \mathrm{~b}$ show the results corresponding to the first static strategy, S1. In figures $2 \mathrm{a}$ and $2 \mathrm{~b}$ the results obtained for different values of $\alpha$ when the bandwidth and class proportionality criteria were applied are shown. Clearly, when bandwidth proportionality is used, the set of cell-edge users becomes smaller leaving more PRBs than the class proportionality case. Because 
of this, the values obtained for fairness are slightly better. On the other hand, class proportionality brings a significant efficiency improvement at the expense of a small fairness degradation. Looking at the effect of $\alpha$, it is evident that an increase of such parameter leads to better values of efficiency no matter which classification criterion has been selected. This means that this parameter can be tuned independently of the number of users of each type.

Fig. 2c shows the results for the static strategy S2. In this scheme, an additional cell area (or class) is introduced in order to obtain a more detailed classification. In this case, class proportionality was applied and the effect of the variations of $\alpha_{1}$ and $\alpha_{2}$ was studied.

Since the users are supposed to be grouped into more classes, fairness is expected to be improved. Nevertheless, static S1 outperforms static S2 in terms of efficiency since the allocation of a wider band to the set of users enjoying a better radio channel becomes the predominant effect, especially when the SINR threshold is shifted to higher values. On the other hand, when we look at the joint effect of $\alpha_{1}$ and $\alpha_{2}$, it is clear that the higher the difference in the power assigned to each class, the higher value of fairness is obtained although with a marginal gain of S2.b over S2.c. The effect on efficiency is the opposite. Since the bandwidth assigned to each class is fixed, as we equalize the power allocated to them, we are taking energy previously assigned to the users having worse channel users and moving it to users with better channel conditions.

Finally, the results corresponding to the static scheme S3 are shown in Fig. 2d and 2e. In this case inter-class interference is completely removed. This is done at the expense of a reduction in the available bandwidth at each cell as it can be seen from Fig. 1c. The advantage of doing so is that higher levels of SINR can be achieved within the cell; this opens the door to the use of techniques requiring good channel estimations, such as spatial multiplexing with a MIMO configuration, in a wider area of the cell with possible throughput improvement. Despite having a reduction of $7 \%$ in terms of spectral efficiency, the effectiveness in the use of resources is higher than the previous cases; this is evident since the number of PRB and bits per $\mathrm{W}$ is significantly greater.

\section{Conclusions}

A fair comparison among different static ICIC strategies has been presented in this work. In order to assess the performance not only from the efficiency-fairness tradeoff but also from the effectiveness in the resources usage perspective, a set of metrics were employed. Moreover, these results were obtained from different experiments with identical simulation conditions.

The main conclusions are summarized as follows:

- In general, a more accurate classification of users does not imply a better performance. In addition, we have seen that the fairness among classes is more affected by the amount of power allocated to each one.

- Based on the results, it is clear that, when a fine tuning of the parameters is done, a very attractive efficiency-fairness tradeoff can be achieved by using 
any of the static ICIC strategies. Nevertheless, there are subtle differences among them. While schemes S1 and S2 can be used in scenarios where the values of SINR are not very good, scheme S3 is clearly the way to follow in the cases when higher order modulations and advanced techniques requiring higher levels of SINR are expected to be used.

- From a practical perspective, static ICIC coordination techniques are a very attractive alternative for operators since there is not complexity associated nor signalling exchange.

- Moreover, it is important to recall that perhaps the most important issue related to static ICIC coordination is the lack of adaptability to the network conditions; these include changing traffic loads, traffic patterns and mobility. Also, fault tolerance is a critic element that could be further investigated.

- Finally, it is of special interest to study the behaviour of such ICIC strategies when channel feedback is prohibitive or not available at all, for instance scenarios with high movility. These among others are future research lines we plan to work on.

\section{References}

[1] 3GPP: TR 25.814 v7.1.0 (Release 7) - Physical layer aspects for Evolved Universal Terrestrial Radio Access (UTRA). Technical Report, 3GPP (2006)

[2] Huawei, G.: 3GPP TSG RAN WG1 Meeting \#41 - Soft Frequency Reuse Scheme for UTRAN LTE. Technical Report available as R1-050507, 3GPP (2005)

[3] Ericsson, G.: 3GPP TSG RAN WG1 Meeting \#45 - Downlink Inter-cell Interference Co-ordination/Avoidance - Evaluation of Frequency Reuse. Technical Report available as R1-061374, 3GPP (2006)

[4] Alcatel, G.: 3GPP TSG RAN WG1 Meeting \#44 - System Simulation Results for Downlink Interference Coordination. Technical Report available as R1-060209, 3GPP (2006)

[5] Zhang, X., He, C., Jiang, L., Xu, J.: Inter-cell Interference Coordination Based on Softer Frequency Reuse in OFDMA Cellular Systems. In: Proc. IEEE Int. Conf. Neural Networks and Signal Processing (ICNNSP 2008), Zhenjiang (China) (June 7 - 11, 2008)

[6] Ruiz, S., Haro, E., González, D., García-Lozano, M., Olmos, J.: Comparison of Different Distributed Scheduling Strategies for Static/Dynamic LTE Scenarios. Technical Report available as TD(09)992, COST 2100, Wien (Austria) (September $28-30,2009)$

[7] Elayoubi, S.E., Haddada, B., Fourestié, B.: Performance Evaluation of Frequency Planning Schemes in OFDMA-based Networks. IEEE Trans. on Wireless Comm. 7(5) (May 2008) $1623-1633$

[8] Corvino, V., Gesbert, D., Verdone, R.: A Novel Distributed Interference Mitigation Technique Using Power Planning. In: Proc. IEEE Wireless Comm. and Networking Conf. (WCNC 2009), Budapest (Hungary) (April 5 - 8, 2009)

[9] Boghe, M., Gross, J., Wolisz, A.: Optimal Power Masking in Soft Frequency Reuse Based OFDMA Networks. In: Proc. European Wireless Conf. (EW 2009), Aalborg (Denmark) (May 17 - 20, 2009)

[10] Jain, R.: The Art of Computer Systems Performance Analysis. First edn. John Wiley \& Sons, New Yotk, USA (1991) 
[11] 3GPP: TR 36.201 v8.2.0 (Release 8) - LTE Physical Layer - General Description. Technical Report, 3GPP (2008)

[12] 3GPP: TR 36.211 v8.5.0 (Release 8) - Physical Channels and Modulation. Technical Report, 3GPP (2008)

[13] 3GPP: TR 36.212 v8.5.1 (Release 8) - Multiplexing and Channel Coding. Technical Report, 3GPP (2008)

[14] Fraile, R., Lázaro, O., Cardona, N.: Two Dimensional Shadowing Model. Technical Report available as TD(03)171, COST 273, Prague (Czech Rep.) (September 24-26, 2003)

[15] Olmos, J., Serra, A., Ruiz, S., García-Lozano, M., González, D.: Exponential Effective SIR Metric for LTE Downlink. In: Proc. IEEE Int. Symp. on Personal, Indoor and Mobile Radio Comm. (PIMRC 2009), Tokyo (Japan) (September 13 $-16,2009)$

[16] Zheng, H., Wu, M., Choi, Y., Himayat, N., Zhang, J., Zhang, S.: Link Performance Abstraction for ML Receivers Based on RBIR Metrics. Technical Report C802.16m-08, IEEE (2008) 


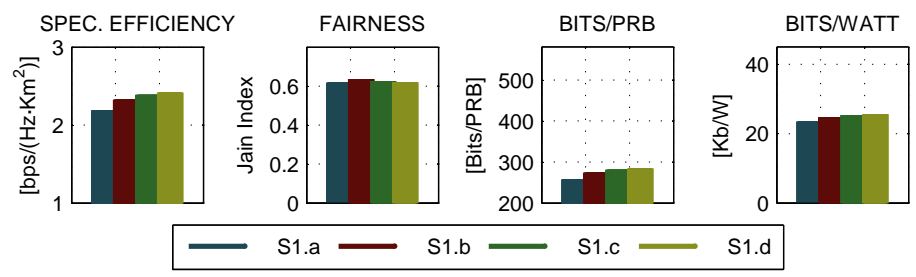

(a) ICIC S1.

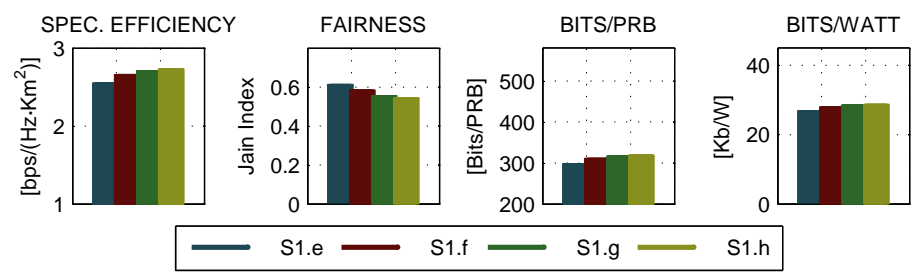

(b) ICIC S1.
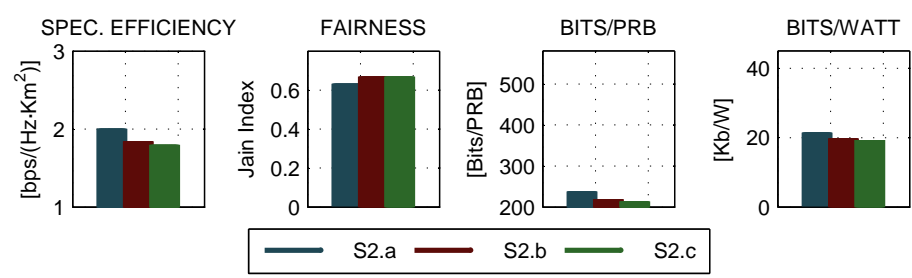

(c) ICIC S2.

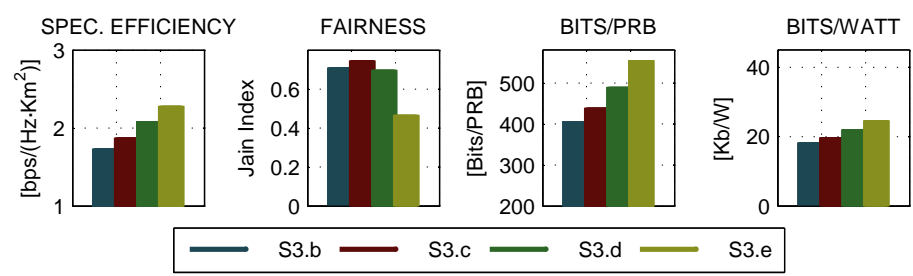

(d) ICIC S3.

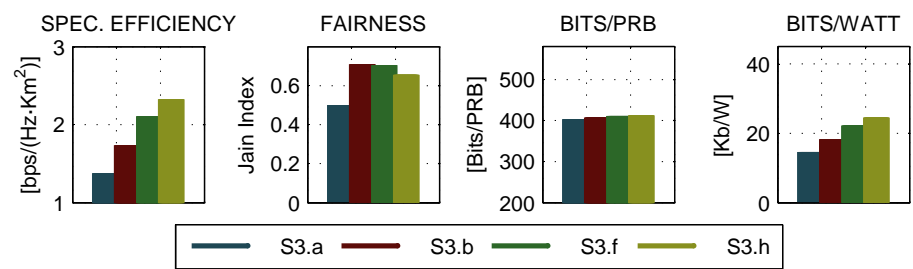

(e) ICIC S3.

Fig. 2. Results obtained for the different ICIC approaches. 